\title{
Artificial Neural Network for Cursive Handwriting Recognition System
}

\author{
Aditi Gupta
}

\begin{abstract}
Cursive Handwriting acknowledgment is an extremely testing zone because of the one of a kind styles of composing starting with one individual then onto the next. Right now, disconnected cursive composing character acknowledgment framework is portrayed utilizing an Artificial Neural Network. The highlights of every character written in the information are extricated and afterward sent to the neural system. Informational collections, having writings of various individuals are utilized in making framework. The suggested acknowledgment framework yields elevated steps of exactness when contrasted with the ordinary methodologies right now. This framework can effectively perceive cursive messages and convert them into auxiliary structure.
\end{abstract}

Keywords: Image Processing, Feature extraction, neural networks, Cursive handwriting, Classification

\section{INTRODUCTION}

Cursive composing acknowledgment is one of the testing fact finding regions in picture preparation and design acknowledgment. Every individual has an alternate style of composing a similar letters in order. There are additional varieties in the content composed by a similar individual periodically. The advancement of cursive composing acknowledgment frameworks has prompted better communication among humans and Technology. Different fact finding have been directed concentrating on noval ideas that target lessening the preparation duration and giving greater exactness. Penmanship acknowledgment can be of two kinds, disconnected and online acknowledgment techniques. In the disconnected methodology, the information is acquired by filtering the content composed on the paper utilizing a pen/pencil as a picture. In the on-line framework the 2D directions of progressive focuses are spoken to as an element of time. The info is subsequently acquired by electric eye gadgets like electronic tablets or digitizers. The online framework acts progressively yet the disconnected methodology can give more elevated steps of precision in perceiving the characters. There are various implementation when these frameworks can be utilized viably like mail arranging, bank preparing, and report perusing and postal location acknowledgment. The initial phase in any hand written acknowledgment framework is preprocessing trailed by division and highlight extraction. Pre-handling is basically to shape the information picture into a structure appropriate for division. In the splitting up process, every character is isolated. Afterwards, every character is resized into $\mathrm{m} \times \mathrm{n}$ pixels towards the preparation grid.

Revised Manuscript Received on June 15, 2020.

* Correspondence Author

Aditi Gupta*, Assistant Professor, Department of Computer Science, DAV College for Boys, Hathi Gate, Amritsar. India.

(C) The Authors. Published by Blue Eyes Intelligence Engineering and Sciences Publication (BEIESP). This is an open access article under the CC BY-NC-ND license (http://creativecommons.org/licenses/by-nc-nd/4.0/)
The most basic factor in accomplishing high acknowledgment execution is the choice of suitable component drawing strategy. The techniques like Template coordinating, Chart portrayal, Projection Histograms, Zoning are broadly utilized. An ANN is utilized in the background to execute arrangement and acknowledgment activity. In the disjointed acknowledgment framework, the neural systems have risen as the quick and solid instruments for arrangement for achieving rising proficiency. Some significant order strategies incorporate measurable techniques dependent on Bayes choice standard, ANNs, SVM and so forth. Paper is sorted out into different segments. The Second Section states a short review of the current strategies that are being suggested right now. The following, Third Section depicts the suggested approach dependent on multidimensional feed forward neural system. Towards the last areas of paper, we break down the consequences of the suggested approach under different circumstances and close the paper.

\section{CURRENT METHOD}

Cursive penmanship acknowledgment has been a territory of enthusiasm of different scientists because of its pertinence in facilitating various assignments of this present reality. Outstanding commitments have prompted advancement of frameworks which are incredibly quick and effective in perceiving the information writings. The acknowledgment of cursive writings dependent on bifurcation of consistent characters in triplets was suggested in 1999. One word was fragmented into triplets. The consequent triplets had two normal letters. Such idea of covering the characters was utilized to accomplish better acknowledgment grades. A cross relationship framework was kept up to follow the availability betwixt the images. A changed quadratic classifier based plan to perceive messages in six distinctive Indian contents was came in 2007. At that time only, the Horizontal/Vertical strokes alongside zoning systems were projected which announced greater effectiveness. However the element drawing procedure right now is mind boggling and tedious. The strategy additionally utilizes diminishing procedure of the characters which prompts the depletion of specific highlights. Neural Networks have ended up being a proficient apparatus for perceiving manually written writings. In 2011, a methodology dependent on the above was suggested where the characters must be of an established size. Highlight extrication module was absent from this framework exhibiting a low acknowledgment precision. One more framework dependent on cross breed HMM was suggested in 2011 to perceive unhindered disconnected writings.

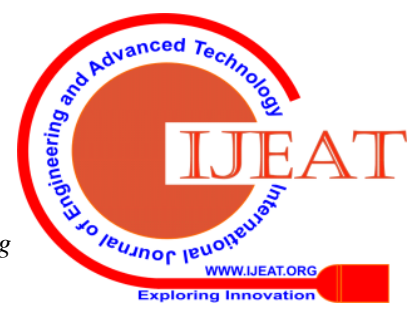




\section{Artificial Neural Network for Cursive Handwriting Recognition System}

The basic piece of the visual model was changed and a Multilayer Perceptron was utilized to perceive the characters. A way to deal with perceive English characters was suggested in 2012. It depended on Fuzzy order hypothesis where an enrollment work was utilized. This capacity depended on the directions (x,y) and the stretch of the character. The level of comparability betwixt the character and prepared picture was utilized to perceive the letter set. A Back Propagation calculation utilizing force thing and job work was recommended in 2013 for cursive composing acknowledgment. The methodology had favorable circumstances like fast pace and better acknowledgment impact.

\section{COMPARISON BETWEEN EXISTING MODELS AND PROPOSED MODEL}

Right now, diagonal include extraction conspire for the acknowledgment of manually written characters is advised. A review of the framework is given in Figure I.

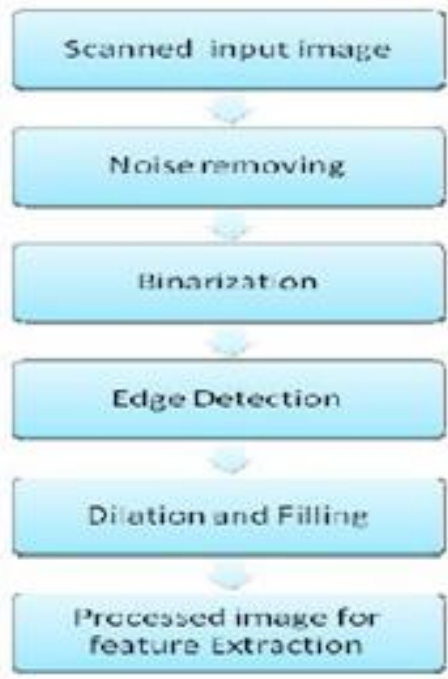

\section{Figure I: Diagram of Cursive Handwriting Reorganization}

In the drawing procedure of features, rescaled singular character of size $80 \mathrm{x} 70$ pixels is additionally separated into 56 equivalent zones, every one of size $10 \times 10$ pixels. The highlights are removed from the pixels of every region progressing along their diagonals. This method is rehashed in every region prompting extrication of 56 highlights for every character. These removed highlights are utilized to prepare a feed forward back proliferation neural system utilized for doing arrangement and acknowledgment assignments. The benefit of the aforesaid system is that it needs smaller duration for preparing the neural system.

\subsection{Image Acquisition}

In Image obtaining, the acknowledgment framework secures an examined picture as an info picture. The picture ought to have a particular arrangement, for example, JPEG, BMT and so forth. The picture is received through a scanner, computerized camera or some other reasonable advanced info gadget.

\subsection{Pre - Processing}

A progression of tasks are executed on the checked information picture. It basically upgrades the picture making it reasonable to divide. The different assignments performed on the picture in pre-handling steps are shown in Figure II. Binarization process changes over a dim scale picture into a parallel picture utilizing an edge strategy. Discovery of boundaries in the binarized picture utilizing sobel administrator, enlarging the picture and stuffing its openings are the activities acted in the last two phases to deliver the already prepared picture appropriate for division. Inclination remedy is likewise done to make accurate the edge of the content.

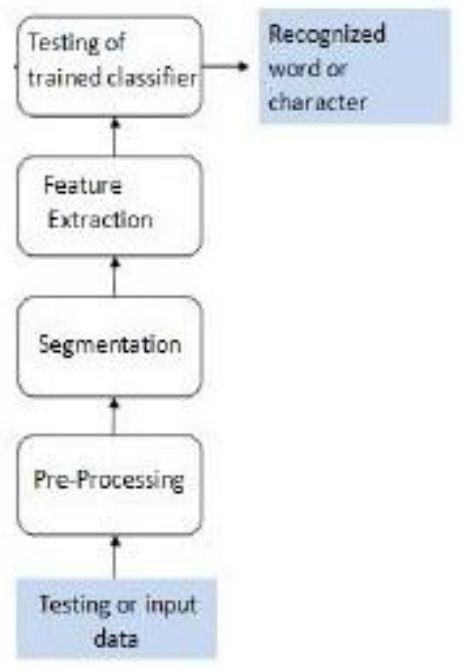

Figure II - Pre-Processing Phase

\subsection{Segmentation}

Right now, portrayal of grouping of characters is divided into various sub portrayals of single character. In the proposed framework, the pre-handled info picture is sectioned into restricted characters by doling out a number to each character using a tagging procedure. This tagging gives data the number of characters in the picture. Every single character is unvarying rescaled into $80 \mathrm{X} 70$ pixels for grouping and acknowledgment organize.

\subsection{Feature Extraction}

Right now, highlights of the characters that are pivotal to group all of those at acknowledgment organize are separated. This stage is very important as its powerful working enhances the acknowledgment grade and diminishes the error in classifications. Askew element drawing conspire for perceiving disconnected transcribed characters is proposed right now. Each character picture of size $80 \times 70$ pixels is separated into 56 equivalent zones, every one of size $10 \times 10$ pixels. The series of codes are utilized for recognizing the headings as appeared in Figure III so as to extricate the highlights of a character. The highlights are separated from every zone pixels by heading along the diagonals of its particular 10X10 pixels.

Published By:

Blue Eyes Intelligence Engineering 
Every zone has 19 slanting lines and the frontal area pixels present along and every corner to corner line is added to get a solitary sub-highlight, therefore 19 sub-highlights are acquired from the every zone. These 19 sub-highlights esteems are arrived at the midpoint of to frame a solitary element esteem and arranged in the specific zone.

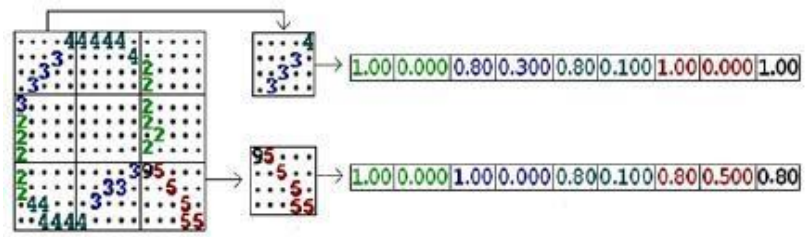

Figure III: Directional Feature of Extraction

This method is successively rehashed for every zones. Few zones could be there whose diagonals are vacant of frontal area pixels. The component esteems relating to these zones are zero. At long last, 56 highlights are removed for all characters. Likewise, 8 and 7 highlights are received by taking an average of the quantities set in zones row wise and column wise, individually. Consequently, each character is spoken to by 78 , features.

\subsection{Identification}

It's a dynamic piece of an acknowledgment framework which uses the highlights removed in the past step. A feed forward back spread neural system as given in Fig. IV, with 2 shrouded layers is utilized to play out the order. The concealed layers use log sigmoid actuation work, and the yield layer is a serious layer, as one of the characters is to be distinguished. The component vector is indicated as $\mathrm{X}$ where $X=\quad(f 1, f 2, \ldots, f d)$ where $f$ means highlights and $\mathrm{d}$ is the quantity of zones into which each character is partitioned.

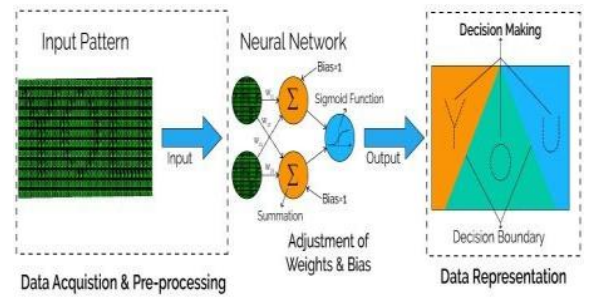

Fig - IV: Architecture of the Neural Network

The quantity of information neurons is controlled by length of the component vector $d$. The all out quantities of characters $\mathrm{n}$ decides the quantity of neurons in the yield layer. The quantity of neurons in the shrouded layers is acquired by experimentation. The most minimized system is picked and introduced.

The suggested framework is being executed utilizing Matlab. A filtered picture is used as set of information and feed forward design is also utilized. The setup of neural system incorporates an info layer with 56 data sources, 2 concealed layers each with 100 neurons and a yield layer having 27 neurons. The system is prepared utilizing the angle plummet back spread strategy with energy and versatile acquiring rate and length arcs exchange work. Neural system is prepared utilizing studied set of data. An acknowledgment framework utilizing two diverse element

\section{TRIAL AND THE FINDINGS}

lengths is fabricated. The quantity of info hubs is picked dependent on the quantity of highlights.

In the wake of preparing the system, the acknowledgment framework was tried utilizing a few obscure dataset and the outcomes acquired are broke down here. Three distinct methods for highlight evocation are utilized for character acknowledgment in the recommended framework i.e. Flat heading, steep course \& corner to corner bearing. The element vector size is picked as 56 , i.e. without row wise and column wise highlights. The outcomes acquired utilizing three unique sorts of highlight extraction are outlined in Table I.

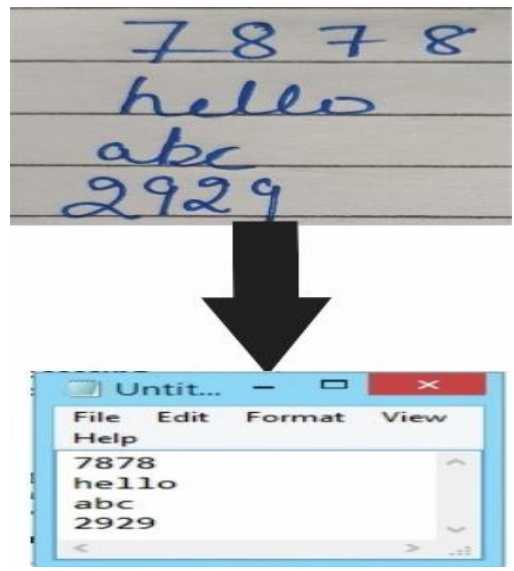

Fig - V: Input Text and Final Result

The touchstone for picking the kind of highlight evocation are: Firstly, the pace of combination, for example count of ages needed to accomplish the preparation objective and secondly, the preparation of steadiness. Be that as it may, the most significant parameter of intrigue is the precision of the acknowledgment framework. An example input picture being changed over into the ideal outcome ie. Perceived characters are being appeared in Figure V.

The outcomes introduced in Table I presents that the slanting component evocation produces great acknowledgment exactness contrasted with the others kinds of highlight extraction. The ideal execution objective is being accomplished in Nine Hundred and twenty three ages.

Table I: Comparison of Recognition Rates obtained with Different Orientations

\begin{tabular}{|l|l|l|l|}
\hline Network & I & II & III \\
\hline $\begin{array}{l}\text { Features } \\
\text { Extraction type }\end{array}$ & Vertical & Horizontal & Diagonal \\
\hline $\begin{array}{l}\text { Number of nodes } \\
\text { in input layer }\end{array}$ & 56 & 56 & 56 \\
\hline $\begin{array}{l}\text { Number of nodes } \\
\text { in 1 } 1^{\text {st }} \text { input layer }\end{array}$ & 100 & 100 & 100 \\
\hline $\begin{array}{l}\text { Number of nodes } \\
\text { in 1 } 1^{\text {nd }} \text { input layer }\end{array}$ & 100 & 100 & 100 \\
\hline $\begin{array}{l}\text { Number of nodes } \\
\text { in output layer }\end{array}$ & 27 & 27 & 27 \\
\hline $\begin{array}{l}\text { Percentage of } \\
\text { Recognition }\end{array}$ & 92.69 & 93.68 & 97.80 \\
\hline
\end{tabular}

V. CONCLUSION

A basic disconnected cursive character acknowledgment framework utilizing another sort of highlight extraction,

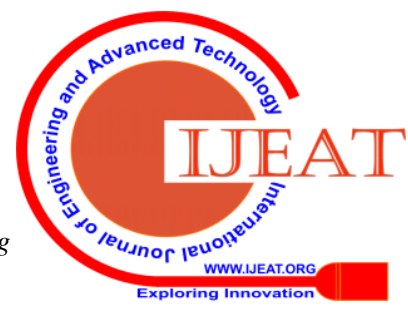




\section{Artificial Neural Network for Cursive Handwriting Recognition System}

in particular, corner to corner include the recommended evocation. The NN used to perceive the characters is manufactured utilizing 56 highlights. Thinking about the acknowledgment productivity of the suggested inclining technique for highlight evocation, the neural system acknowledgment framework is prepared utilizing the flat and steep element evocation strategies, six distinctive acknowledgment systems are fabricated.

From the test outcomes it is distinguished that the corner to corner strategy for highlight evocation gives the most elevated acknowledgment precision of upto 97\%. The inclining technique for highlight extraction is confirmed utilizing various test pictures. The proposed disconnected written by hand character acknowledgment framework with higher value acknowledgment rates will be famously reasonable for a few implementation including postal/package address acknowledgment, bank preparing, record perusing \& transformation of a random written archive into basic content structure.

\section{REFERENCES}

1. F. Kimura, T. Wakabayashi, U. Pal, “ Handwritten Numeral Recognition of six Popular Scripts ", Ninth

2. International Conference on Document Analysis and Recognition, Vol. 2, pp. $749-753,2007$.

3. M. Patel and S. P. Thakkar, "Handwritten Character Recognition in English - A Survey ", International

4. Journal of Advanced Research in Computer and Communication Engineering, Vol. 4 - No. 2, pp. 345 - 350, 2015.

5. M. N. Mangoli and Prof. S. Desai, “ Optical Character Recognition for Cursive Handwriting ”, International

6. Research Journal of Engineering and Technology, Vol. 3- No. 5, pp. 792 - 795, 2016.

7. N. yadav and P. Yadav, “ Handwriting Recognition System - A Review ", International Journal of Computer

8. Applications, Vol. 114 - No. 19, pp. 36 - 40, 2015

9. B. Kumar, N. Sharma and T. Patnaik, " Recognition for Handwritten English Characters : A Review",International Journal of Engineering and Innovative Technology, Vol. 2 - No. 7, pp. 318 321, 2013.

10. Dr. S. Upadhyay and P. N. Tushar, "Chain Code Based Handwritten Cursive Character Recognition System with Better Segmentation using Neural Network ", International Journal of Computational Engineering Research, Vol. 3 - No. 5, pp. 60 - 63, 2013.

11. A. Bhushan, J. Supriya and P. Kalyani, " Handwritten Script Recognition ", IOSR Journal of Computer

12. Engineering, pp. $30-33,2012$.

13. A. Pall and D. Singh, "Handwritten English Character Recognition Using Neural Network ", International

14. Journal of Computer Science and Communication, Vol. 1- No. 2, pp. $141-144,2010$.

15. D. Acharya, N. V. Reddyand Krishnamurthy, “ Isolated Handwritten Kannada Numeral Recognition using Structural feature and K means Cluster ", InternationalJournal of Information Technology and Knowledge Management, pp. 125 - 129, 2009.

16. Utkarsh Dwivedi, Pranjal Rajput, Manish Kumar Sharma ,"Cursive Handwriting Recognition System Using Feature Extraction and Artificial Neural Network “ ,International Research Journal of Engineering and Technology

\section{AUTHOR PROFILE}

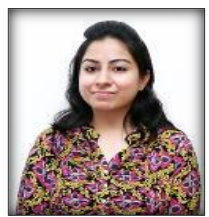

Aditi Gupta, Assistant Professor Dept. of Computer Science, DAV College for Boys, Hathi Gate, Amritsar. Qualification: M.Tech, B.Tech, UGC-Net\& JRF Cleared. Area of interest: Big Data, Artificial Neural Network, Machine Learning. Number of Research. Papers already published: 07

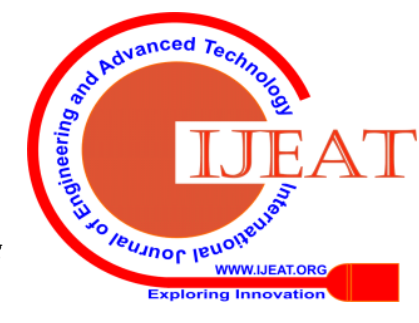

